\title{
Robust Face Image Matching under Illumination Variations
}

\author{
Chyuan-Huei Thomas Yang \\ Department of Computer Science, National Tsing Hua University, 101 Kuang Fu Road, Section 2, Hsinchu 300, Taiwan \\ Email: chyang@cs.nthu.edu.tw \\ Shang-Hong Lai \\ Department of Computer Science, National Tsing Hua University, 101 Kuang Fu Road, Section 2, Hsinchu 300, Taiwan \\ Email:lai@cs.nthu.edu.tw

\section{Long-Wen Chang} \\ Department of Computer Science, National Tsing Hua University, 101 Kuang Fu Road, Section 2, Hsinchu 300, Taiwan \\ Email:lchang@cs.nthu.edu.tw
}

Received 1 September 2003; Revised 21 September 2004

\begin{abstract}
Face image matching is an essential step for face recognition and face verification. It is difficult to achieve robust face matching under various image acquisition conditions. In this paper, a novel face image matching algorithm robust against illumination variations is proposed. The proposed image matching algorithm is motivated by the characteristics of high image gradient along the face contours. We define a new consistency measure as the inner product between two normalized gradient vectors at the corresponding locations in two images. The normalized gradient is obtained by dividing the computed gradient vector by the corresponding locally maximal gradient magnitude. Then we compute the average consistency measures for all pairs of the corresponding face contour pixels to be the robust matching measure between two face images. To alleviate the problem due to shadow and intensity saturation, we introduce an intensity weighting function for each individual consistency measure to form a weighted average of the consistency measure. This robust consistency measure is further extended to integrate multiple face images of the same person captured under different illumination conditions, thus making our robust face matching algorithm. Experimental results of applying the proposed face image matching algorithm on some well-known face datasets are given in comparison with some existing face recognition methods. The results show that the proposed algorithm consistently outperforms other methods and achieves higher than $93 \%$ recognition rate with three reference images for different datasets under different lighting conditions.
\end{abstract}

Keywords and phrases: robust image matching, face recognition, illumination variations, normalized gradient.

\section{INTRODUCTION}

Face recognition has attracted the attention of a number of researchers from academia and industry because of its challenges and related applications, such as security access control, personal ID verification, e-commerce, video surveillance, and so forth. The details of these applications are referred to in the surveys $[1,2,3]$. Face matching is the most important and crucial component in face recognition. Although there have been many efforts in previous works to achieve robust face matching under a wide variety of different image capturing conditions, such as lighting changes, head pose or view angle variations, expression variations, and so forth, these problems are still difficult to overcome. It is a great challenge to achieve robust face matching under all kinds of different face imaging variations. A practical face recognition system needs to work under different imaging conditions, such as different face poses, or different illumination conditions. Therefore, a robust face matching method is essential to the development of an illumination-insensitive face recognition system. In this paper, we particularly focus on robust face matching under different illumination conditions.

Many researchers have proposed face recognition methods or face verification systems under different illumination conditions. Some of these methods extracted representative features from face images to compute the distance between these features. In general, these methods can be categorized into the feature-based approach $[4,5,6,7,8,9,10,11]$, the appearance-based approach $[12,13,14,15,16,17,18,19,20$, $21,22,23]$, and the hybrid approach $[22,24]$. 
In the feature-based approach, it requires the extraction of the face feature points robust against illumination variations. Extracted face edge maps are then compared based on holistic similarity measures, such as the Hausdorff distance [8]. Many methods have been presented for robust feature point extraction from face images. For example, attention points are selected as the feature points through the analysis of the outputs of the Gabor-filtered images [5]. Points of maximum curvature or inflection points of the shape of the image function were used as the face feature points in [4]. For the comparison of edge maps, an affine coordinate based reprojection framework was proposed to match dense point sets between two input face images of the same individual in [7]. Hsu and Jain [6] built a generic facial model by using a facial measurement in a global-to-local way, and then matched the facial features, such as eyes, nose, mouth, chin, and face border, in both images. In addition, Zhu et al. [11] modeled the lighting change as a local affine transformation of the pixel value with a lowpass filter for the illumination correction.

In the appearance-based face recognition, the eigenface approach was very popular in the past decade. To alleviate the illumination variation problem, it is common to ignore some of the most dominant principal components in the eigenface and Fisherface matching [13] due to their strong relationship with illumination variations. Yang et al. [22] used the kernel PCA, a generalization of classical PCA, to better describe the face space in a nonlinear fashion. Adini et al. [12] reviewed several operations to deal with illumination changes, such as edge maps, 2D Gabor filtering, and image derivatives. The features computed from the Gabor-filtered face images are robust against illumination variations [18]. In [14], principal component analysis is combined with Gabor filtering for face recognition. Recently, Georghiades et al. $[15,16]$ proposed a new approach to comparing face images under different illumination conditions by introducing an illumination cone constructed from several images of the same person captured at the same pose under different illumination directions. Moghaddam et al. [20] proposed a probabilistic measure of similarity based on Bayesian (MAP) analysis of image differences for image matching. They showed the superior performance of this matching method over the standard Euclidean nearest-neighbor eigenface matching method through experiments.

In the hybrid approach, face recognition is achieved by using a face model consisting of face shape as well as image intensity information. For example, an active appearance model (AAM), which is a statistical model of shape and of grey-level appearance, was proposed to model face images [24]. In addition, Wiskott et al. [25] formulated the face recognition problem as elastic bunch graph matching. They represented the face by label graphs based on the Gabor transform and matched faces via an elastic graph matching process. Furthermore, Zhao and Chellappa [23] developed a shape-based face recognition system through an illumination-independent ratio image derived from applying symmetric shape from shading to face images.
In this paper, we propose a novel method for robust face image matching under different illumination conditions. We define locally normalized gradient vectors and a consistency measure between normalized gradient vectors. We accumulate the consistency measure with appropriate weighting to define a new matching score between images. Then, this matching score is generalized to include multiple reference face image to improve its robustness. The rest of this paper is organized as follows. We describe the proposed robust face matching method in Section 2. In Section 3, we show some experimental results of applying the proposed method on three well-known face databases to demonstrate the accurate performance of the proposed algorithm over some previous methods. Finally, some conclusions are given in Section 4.

\section{ROBUST FACE MATCHING METHOD}

In this section we present the proposed robust face image matching algorithm, which is based on the consistency between the normalized gradients at corresponding points along the face contours. We first present the robust face image matching algorithm with one reference face image. Then, this algorithm is extended to include multiple reference images of the same person. Note that we assume all the face images for comparison are at the same face pose, since the main goal of this paper is to achieve robust face image matching under different lighting conditions. Although face-pose variation is another major problem in face recognition, we only focus on face image matching under different illumination conditions in this paper. We assume there is no face-pose variation between the face images in comparison. In the following, we are going to describe our proposed algorithm in detail.

The proposed robust face matching approach is based on the assumption that the edge contours of face images are distributed similarly under different illumination conditions. Let a face image be denoted by $I$, and the face edge contour is extracted from a prototype face image by standard edge detection and stored in a set $\Gamma$. When the face images and the corresponding face contours are of the same person at the same pose, it is intuitive to assume that the contour integral of the gradient magnitude of one face image at the properly transformed face contour locations determined from another face image is maximal. The geometric transformation is required to describe the matching between two face images. The geometric transformation of the pixel coordinate $(i, j)$, represented by $T$, considered in this paper consists of $2 \mathrm{D}$ translation, rotation, and scaling. It can be written as

$$
T_{(\rho, \theta, \Delta x, \Delta y)}(x, y)=\rho\left(\begin{array}{cc}
\cos \theta & -\sin \theta \\
\sin \theta & \cos \theta
\end{array}\right)\left(\begin{array}{l}
x \\
y
\end{array}\right)+\left(\begin{array}{l}
\Delta x \\
\Delta y
\end{array}\right)
$$

where $\rho$ is the scaling parameter, $\theta$ is the rotation angle, $\Delta x$ is the $x$-axis translation, and $\Delta y$ is the $y$-axis translation. Let the vector $\mathbf{p}$ denote the collection of all these geometric transformation parameters, that is, $\mathbf{p}=(\rho, \theta, \Delta x, \Delta y)$. A cumulative contour gradient measure based on the above idea 
is given as follows:

$$
E(\mathbf{p}, I)=\sum_{i, j \in \Gamma}\left|\nabla I\left(T_{\mathbf{p}}(i, j)\right)\right| .
$$

When the above cumulative gradient measure is used for face matching, it is susceptible to errors under different lighting conditions. To account for the illumination variation problem, we use a relative gradient magnitude to substitute the previous absolute gradient. The relative gradient magnitude is obtained by dividing the absolute gradient by the local maximal absolute gradient at the current location. This leads to the following normalized contour gradient measure:

$$
E(\mathbf{p} ; I)=\frac{1}{|\Gamma|} \sum_{(i, j) \in \Gamma} \frac{\left|\nabla I\left(T_{\mathbf{p}}(i, j)\right)\right|}{\max _{(k, l) \in W_{T_{\mathbf{p}}(i, j)}}|\nabla I(k, l)|+c},
$$

where $W_{T(i, j)}$ is the local window centered at the transformed location $T(i, j)$ and $c$ is a positive constant to be used to suppress noise amplification for the area with all pixels of very small gradients. The symbol $|\Gamma|$ denotes the total number of pixels in the set $\Gamma$.

To make sure that the extracted contour locations contain the largest locally relative gradient magnitudes, the edge detection used for contour extraction is accomplished by selecting the candidate edge locations with local maximum of gradient magnitudes along its gradient direction in a local neighborhood. Thus, the contour should be consistent with the locations of the greatest relative gradients. In the above normalized contour gradient measure, we only consider the magnitude of the image gradient and ignore the direction of the gradient vector. To make the image matching more robust, we include the orientation consistency of gradient vectors into the above measure to form a gradient consistency. Thus, the normalized consistency measure between two images, called image similarity measure, is modified as follows:

$$
\begin{aligned}
& E\left(\mathbf{p} ; F, I_{0}\right)=\frac{1}{|\Gamma|} \sum_{(i, j) \in \Gamma} \mid \frac{R_{\theta}\left(\nabla I_{0}(i, j)\right)}{\max _{(k, l) \in W_{(i, j)}}\left|\nabla I_{0}(k, l)\right|+c} \\
& \bullet \frac{\nabla F\left(T_{\mathbf{p}}(i, j)\right)}{\max _{(k, l) \in W_{T_{\mathbf{p}}(i, j)}}|\nabla F(k, l)|+c} \mid,
\end{aligned}
$$

where $I_{0}$ is the template image, the sample image in the face database for training, $F$ is the input image containing a face to be matched, $R_{\theta}$ is the $2 \mathrm{D}$ rotation operator with rotation angle $\theta$ specified in the parameter vector $\mathbf{p}$, and the symbol - denotes the inner product. The inclusion of the rotation operator in the consistency measure between two normalized gradient vectors is to compensate for the discrepancy between the corresponding gradient vectors caused by the rotation between the two images. Since the absolute value of the normalized inner product is between 0 and 1 , the above normalized similarity measure is also between 0 and 1 . The larger the value, the more similar the input face image is to the template face image. If the normalized similarity measure is one, then these two face images in comparison are the completely same.

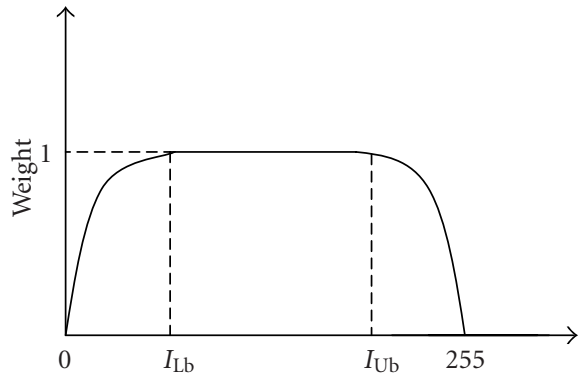

FIgURE 1: The intensity weighting function.

To alleviate the problem due to shadow or intensity saturation, we assign smaller weight in the individual similarity measures for points with very bright or very dark intensity values. Thus, the modified similarity measure becomes

$$
\begin{aligned}
& E^{\prime}\left(\mathbf{p} ; F, I_{0}\right)=\sum_{(i, j) \in \Gamma} \mid \frac{R_{\theta}\left(\nabla I_{0}(i, j)\right)}{\max _{(k, l) \in W_{(i, j)}}\left|\nabla I_{0}(k, l)\right|+c} \\
& \cdot \frac{\nabla F\left(T_{\mathbf{p}}(i, j)\right)}{\max _{(k, l) \in W_{\mathbb{T}_{\mathbf{p}}(i, j)}|\nabla F(k, l)|+c} \mid} \\
& \times \tau\left(F\left(T_{\mathbf{p}}(i, j)\right)\right)\left(\sum_{(i, j) \in \Gamma} \tau\left(F\left(T_{\mathbf{p}}(i, j)\right)\right)\right)^{-1},
\end{aligned}
$$

where $\tau$ is the intensity weighting function given by

$$
\tau(I)= \begin{cases}\sin \left(\frac{\pi}{2} * \frac{I}{I_{\mathrm{Lb}}}\right), & 0 \leq I<I_{\mathrm{Lb}}, \\ 1, & I_{\mathrm{Lb}} \leq I \leq I_{\mathrm{Ub}}, \\ \cos \left(\frac{\pi}{2} * \frac{I-I_{\mathrm{Ub}}}{255-I_{\mathrm{Ub}}}\right), & I_{\mathrm{Ub}}<I \leq 255,\end{cases}
$$

where $I_{\mathrm{Lb}}$ and $I_{\mathrm{Ub}}$ mean the lower bound and the upper bound of the weight function. This weighting function is illustrated in Figure 1. For pixels with intensity values closer to zero or 255, we assign smaller weights to their contributions to the similarity measure. The normalization factor in the denominator of (5) is the sum of all the weights at the transformed locations. With the use of this normalization factor, this modified similarity measure is normalized into the interval $[0,1]$.

We extend the face image matching based on the consistency measure of the normalized gradients between two images to allow for using multiple reference face images. This extension is used to improve the robustness against illumination variations. We assume that there are multiple face reference images of the same person captured at the same pose with different lighting conditions. These images are denoted by $\mathbf{I}_{1}, \mathbf{I}_{2}, \ldots, \mathbf{I}_{\mathrm{N}}$, respectively. In Section 1 , the similarity measure of normalized gradients between two images is developed and given in (5). We generalize the previous similarity measure by using the best of the individual consistency measure values between the input face image and each of 
the multiple reference face images as follows:

$$
\begin{aligned}
& E^{\prime}\left(\mathbf{p} ; F, I_{n}, n=1, \ldots, N\right) \\
& =\sum_{(i, j) \in \Gamma} \max _{p=1,2,3, \ldots, n} \mid \frac{R_{\theta}(\nabla F(i, j))}{\max _{(k, l) \in W_{(i, j)}}|\nabla F(k, l)|+c} \\
& \quad \frac{\nabla I_{n}\left(T_{\mathbf{p}}(i, j)\right)}{\max _{(k, l) \in W_{T_{\mathbf{p}}(i, j)}}\left|\nabla I_{n}(k, l)\right|+c} \mid \\
& \quad \times \tau\left(F\left(T_{\mathbf{p}}(i, j)\right)\right)\left(\sum_{(i, j) \in \Gamma} \tau\left(F\left(T_{\mathbf{p}}(i, j)\right)\right)\right)^{-1} .
\end{aligned}
$$

In the training of our face image matching algorithm, we extract face edge contours by edge detection with nonmaximal suppression [26] for each of the template face images in the face database. In our face matching method, we extract face contours by edge detection with nonmaximal suppression for each of the template face images in the face database. In addition, we also compute the normalized gradients for the template face images in the database. Then, we compare the input face image $F$ with the set of the reference face images for each candidate by optimizing the following energy function with respect to the geometric transformation parameter vector p:

$$
\max _{\mathbf{p}} E^{\prime}\left(\mathbf{p} ; F, I_{n}, n=1, \ldots, N\right)
$$

where $I_{n}$ is the $n$th face template image for a candidate in the database. This optimization problem can be solved by the Levenberg-Marquardt (LM) algorithm [27] when a good initial guess of the geometric transformation parameters is available. This process can be combined with a face detection algorithm to find the approximate location and size of the face in the input image, thus providing good initial guesses of the geometric transformation parameters. Then, the LM algorithm is applied to maximize the similarity measure function for all the template face images.

The template face with the highest similarity measure after the optimization is closest to the input face. Therefore, it is the result of the nearest-neighbor face recognition. In other words, the face recognition can be formulated as the following optimization problem:

$$
\arg \max _{p \in P} \max _{T} E^{\prime}\left(\mathbf{p} ; F, I_{n}^{(p)}, n=1, \ldots, N\right),
$$

where $I_{n}^{(p)}$ is the $n$th face training image of the $p$ th candidate and $P$ denotes the set of all the candidates in the database. The overall flow diagram of the proposed face recognition method is shown in Figure 2.

\section{EXPERIMENTAL RESULTS}

The results of testing the proposed method on three wellknown benchmarking face databases are reported and compared with those of some existing face recognition methods, including the image derivative, 2D Gabor-filtering, eigenface, and Fisherface-based matching methods. We first investigate

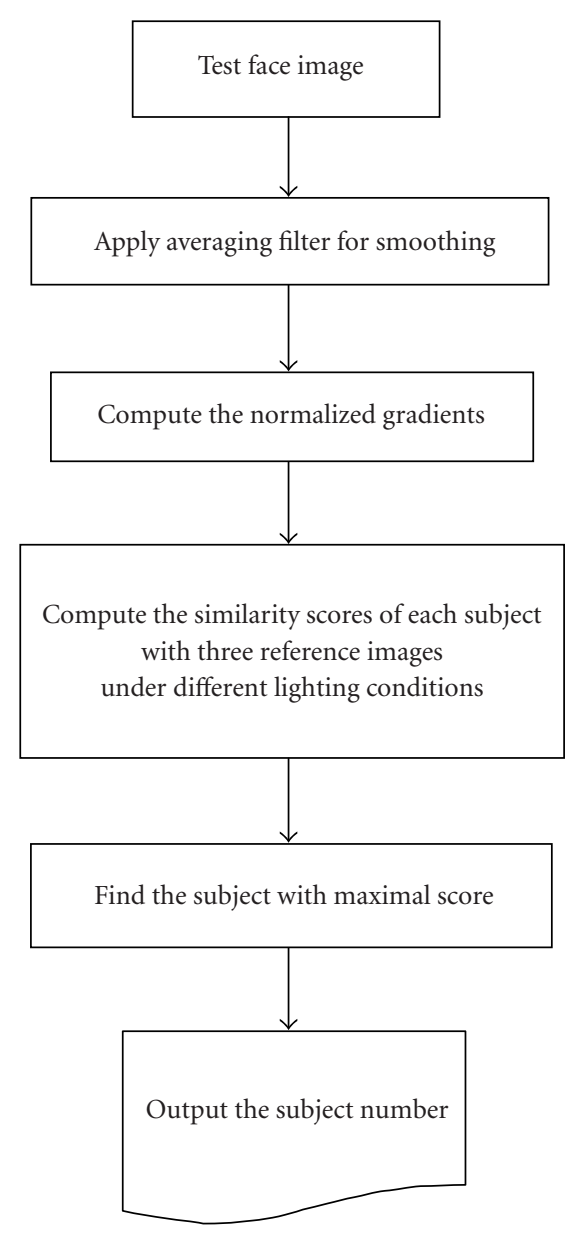

Figure 2: The procedure of the proposed method.

the experimental results of these methods with one reference image on the small Yale Face Database. Then, the experimental results on two larger face databases, namely, Yale Face Database B and CMU PIE Face Database, are given in comparison with the aforementioned face recognition methods. We show our experimental results in the three databases as follows.

\subsection{Tests results on Yale Face Database}

The Yale Face Database $[13,15]$ was used to examine the robustness of the proposed face matching algorithm against lighting changes with only one reference image. It contains 15 subjects captured under three different light conditions; namely, center light, right light, and left light. Examples of one subject in the Yale Face Database under the three different lighting conditions are shown in Figure 3. In our implementation, we applied a smoothing operator on the face images before computing the image gradient. This smoothing operation not only reduces the noise effect but also spreads out the support of the gradient function around contour locations. This helps to increase the convergence region in the optimization problem. We used an averaging operator for smoothing in our implementation for simplicity in implementation. 


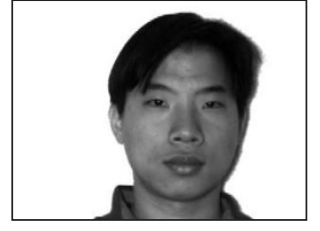

(a)

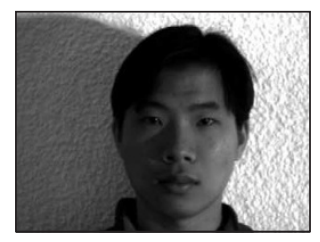

(b)

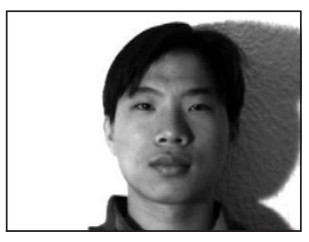

(c)

FIgURE 3: A face set of one subject in the Yale Face Database with (a) center light, (b) right light, and (c) left light.

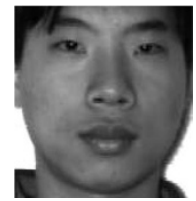

(a)

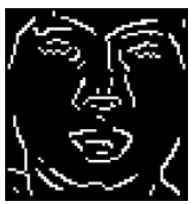

(b)
FIGURe 4: (a) A template image and (b) the extracted face contour map.

There are several tunable parameters in our implementation, such as the mask size for averaging filter, the window size for finding the local maximum, the threshold for edge detection, the lower bound $\left(I_{\mathrm{Lb}}\right)$ and upper bound $\left(I_{\mathrm{Ub}}\right)$ of the weighting function, and the constant $c$ in the similarity measure. For saving the computation time, we downsampled the face image to a quarter of the original size first. We used a $3 \times 3$ average filter, and a $5 \times 5$ local window for gradient normalization. We selected the threshold of the edge detection adaptively based on the percentage cutoff in the histogram of the gradient magnitudes computed from the face image. In our experiments, the lower bound and upper bound of the intensity weighting function were set to 60 and 230, respectively. The constant $c$ was set to 5 . Figure 4 depicts a template face image and the extracted contour of this face template. Figure 5 shows the matching results of the face images in Figure 3 under three different lighting conditions with the face template in Figure 4.

The recognition rate obtained by using the proposed face matching algorithm with one reference face image on this Yale Face Database is $93.33 \%$. Table 1 shows the recognition rates of the proposed method and some other methods through face image matching by using the center-light face image as the reference image. Here the matching methods considered for comparison include the gray-level derivatives method, the 2D Gabor-filter based method, the eigenface method, and the Fisherface method. The gray-level derivative matching method is based on comparing the isotropic derivative image at different scales. The Gabor-filter based matching method compares the Gabor-filtered images at several resolutions. The eigenface method uses principal component analysis (PCA) for reducing the dimensionality to get the projection directions. The Fisherface method computes the features based on Fisher linear discriminant (FLD) to maximize the ratio of between-class scatter to
TABLE 1: The recognition rate of the proposed method and the other methods with one reference face image.

\begin{tabular}{lc}
\hline Method & Recognition rates \\
\hline Gray-level derivative method & $68.18 \%$ \\
2D Gabor-filter method & $81.82 \%$ \\
Eigenface & $56.66 \%$ \\
Fisherface & $86.66 \%$ \\
Proposed method with center light & $93.33 \%$ \\
\hline
\end{tabular}

that of within-class scatter. From Table 1, we can see that the proposed robust face matching algorithm outperforms other methods in terms of recognition accuracy on this dataset.

\subsection{Tests results on Yale Face Database B}

We tested our proposed method on the Yale Face Database B [15] with one and multiple reference images. For the experiments with multiple reference images, we used only three face images at very different lighting conditions. This face database contains 5760 single light source images for 10 subjects (persons). The size of each image is $640 \times 480$. There are 576 images acquired at different poses and with different lighting conditions for each subject. There are 9 different face poses combined with 64 different illumination conditions for each subject. Figure 6 shows the 10 subjects from the Yale Face Database B.

In this paper we focus on the problem of illumination variations with fixed face pose. We used the face images at frontal face pose with different lighting conditions in the Yale database $\mathrm{B}$ to be our experimental dataset. In our experiment, we selected three images "yaleB01_P00A + 000E + 00.bmp," "yaleB01_P00A - 050E + 00.bmp," and "yaleB01_P00A + 050E + 00.bmp" as our multiple reference images, which correspond to the frontal pose with lighting sources from center, left (50 degrees), and right (50 degrees), respectively, as depicted in Figure 7 . Since this database provides the coordinates of eyes for all face images, we select the face regions with proper size from these reference images to be our matching templates. This means these templates are aligned based on the labeled facial feature locations. Figure 8 shows the face template images of the first subject. Figure 9 shows the 36 face images under different lighting conditions for the same subject as the test images. The total number of test images is 360 . 


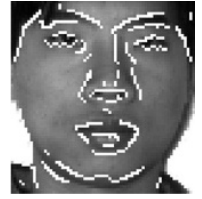

(a)

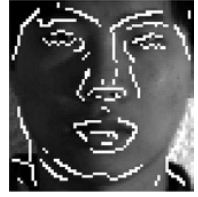

(b)

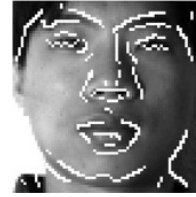

(c)

FIGURE 5: Face image matching results with one of the face template contours overlaid on the input face images under (a) center light, (b) right light, (c) left light conditions are shown.
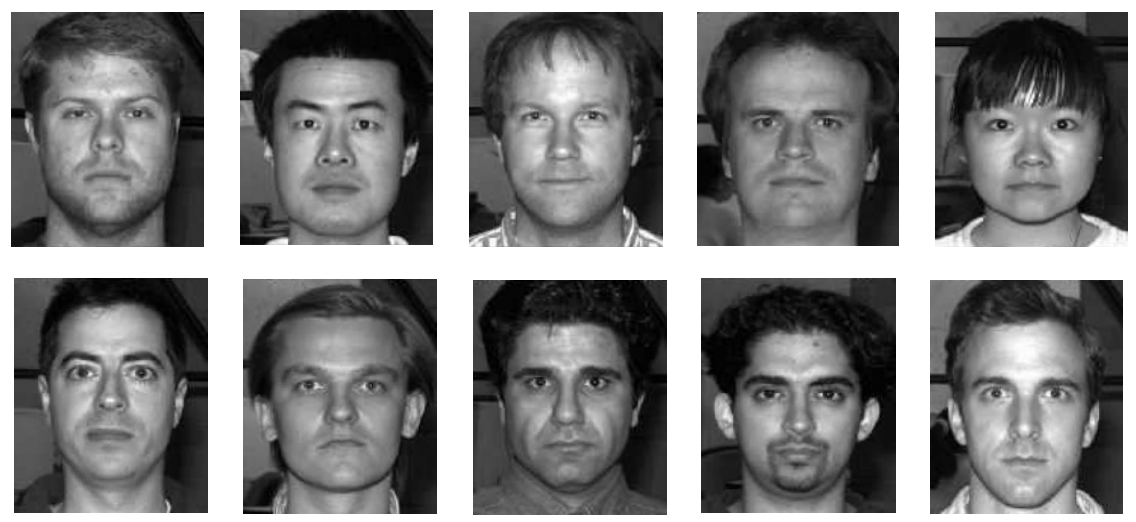

Figure 6: Ten subjects of the Yale Face Database B.

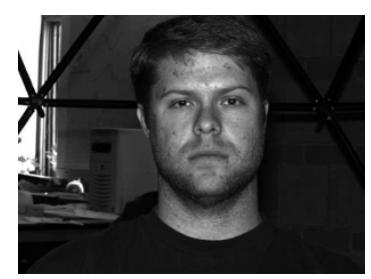

(a)

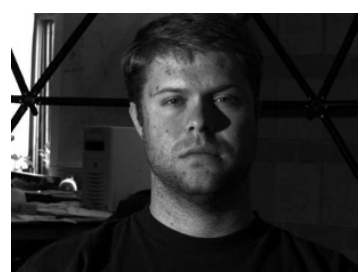

(b)

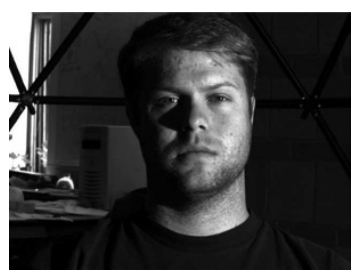

(c)

Figure 7: The original of three reference images of the first subject. (a) YaleBP00A+000E+00, (b) YaleBP00A-050E+00, (c) YaleBP00A+050E+00.

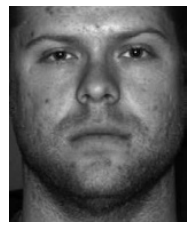

(a)

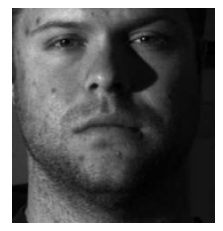

(b)

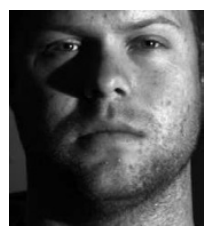

(c)

Figure 8: Three reference images of the first subject from Figure 4: (a) center light, (b) left light, (c) right light.

For reducing the computational time, we downsampled the face image to $1 / 16$ of the original size first. We used the same pre-processing procedure and parameter setting as those described in Section 3.1. Figure 10 shows the matching results of the images for the first and the second subjects with the edge contour of the first subject.
For a reasonable range of light source directions, we select the light directions with the angle between $+/-70$ degrees in the azimuth angle and $+/-70$ degrees in the elevation angle. The total number of images under different lighting conditions for each subject is 39 in our experiments. The recognition rate obtained by using our face matching algorithm 

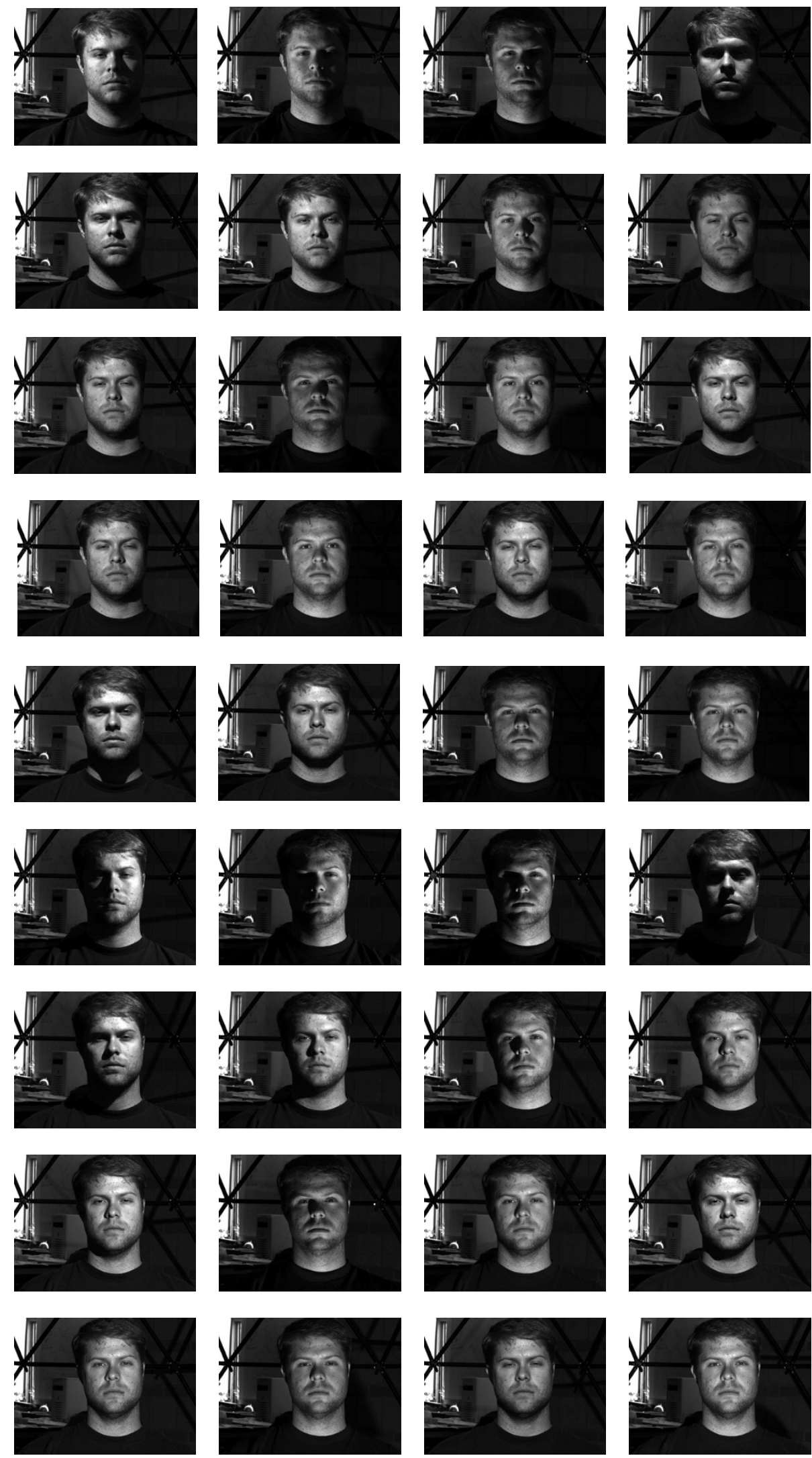

FIgURE 9: The 36 test images with different illumination conditions of the first subject. 


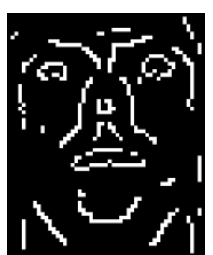

(a)

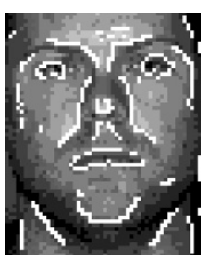

(b)

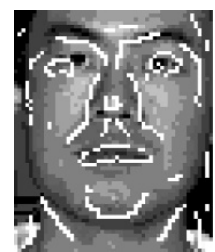

(c)

FIgURE 10: (a) The edge contour of the first subject, (b) the edge contour of the first subject to match itself, and (c) the edge contour of the first subject to match the second subject.

TABLE 2: Comparison of recognition rates by using isotropic graylevel derivatives, 2D Gabor filter, eigenface, Fisherface, and the proposed robust image matching algorithm on the Yale Face Database B.

\begin{tabular}{|c|c|c|}
\hline \multirow[b]{2}{*}{ Methods } & \multicolumn{2}{|c|}{ Reference images } \\
\hline & $\begin{array}{c}\text { One reference } \\
\text { image }\end{array}$ & $\begin{array}{c}\text { Three reference } \\
\text { images }\end{array}$ \\
\hline Isotropic derivatives method & $47.11 \%$ & $57.14 \%$ \\
\hline 2D Gabor-filter method & $62.87 \%$ & $71.33 \%$ \\
\hline Eigenface & $80.26 \%$ & $91.66 \%$ \\
\hline Fisherface & $71.81 \%$ & $88.61 \%$ \\
\hline Proposed method & $78.16 \%$ & $93.95 \%$ \\
\hline
\end{tabular}

with one and three reference images on this test database is shown in Table 2. The average recognition rate is $78.16 \%$ for our method with one reference face image. By using the three reference images in our method, we can achieve $93.95 \%$ recognition rate under different lighting conditions. It is obvious that the proposed face image matching algorithm with multiple reference images has improved the recognition rate significantly from the experimental results.

We also compare the proposed algorithm with the previous methods on this dataset. For a fair comparison, we modified those previous four image matching methods to three reference images to improve their recognition rates. Table 2 shows the recognition rates of all the aforementioned methods with one and three reference images on Yale Database B. The proposed robust image matching algorithm outperforms all the other methods on this dataset for the case with three reference images. Note that the Fisherface algorithm is less accurate than the eigenface method in this experiment, though normally the Fisherface algorithm outperforms the eigenface method [13]. This may be due to the small training data size in this experiment since there are only 10 subjects in this dataset.

\subsection{Tests results on CMU PIE Database}

In this section we show the experimental results on a larger CMU PIE Face Database [28]. We used the CMU PIE illumination database, which contains 1407 face images of 67 people captured under 21 different illumination conditions with frontal face without room light. The size of each im- age is $640 \times 486$. Figure 11 shows all 18 different illumination conditions of the test images of a subject. Such images of one subject are named 27_02,27_03, .., 27_22. The CMU Database consists of color images, but we converted all color images into gray-level images first. In our experiment, we selected the 27_10,27_11, and 27_13 of each subject as the three reference images, as depicted in Figure 12. The templates of this subject in Figure 12 are shown in Figure 13. The rest 1206 images were used for test images. The implementation parameters are the same as those used in the experiment on Yale Face Database B. Figure 14 shows the results of contour matching by using the proposed method for different face images.

The recognition rates obtained by using our face matching algorithm with three reference images on this test database are shown in Table 3. By using the three reference images in our method, we can achieve $94.78 \%$ recognition rate under different lighting conditions. It is evident from Table 3 that the proposed face recognition algorithm outperforms the other methods in terms of recognition accuracy on this dataset.

\section{CONCLUSIONS}

A novel illumination-insensitive robust face matching method was proposed in this paper. This method is based on a new weighted normalized consistency measure of normalized gradients at corresponding points in face images. This new consistency measure is generalized to include multiple face templates of the same person captured under different illumination conditions to improve the robustness. We formulate face recognition problems as an optimization problem of face matching based on the proposed similarity measure. The computational cost of the proposed algorithm compared to that of the area-based image matching method is very low since our similarity measure is computed only at the face contour locations. Experimental results of applying the proposed face image matching algorithm and some existing methods on some benchmarking face datasets were given to demonstrate its superior performance. The results show that the proposed algorithm consistently outperforms other methods and achieves higher than $93 \%$ recognition rate with three reference images for different datasets under different lighting conditions. 

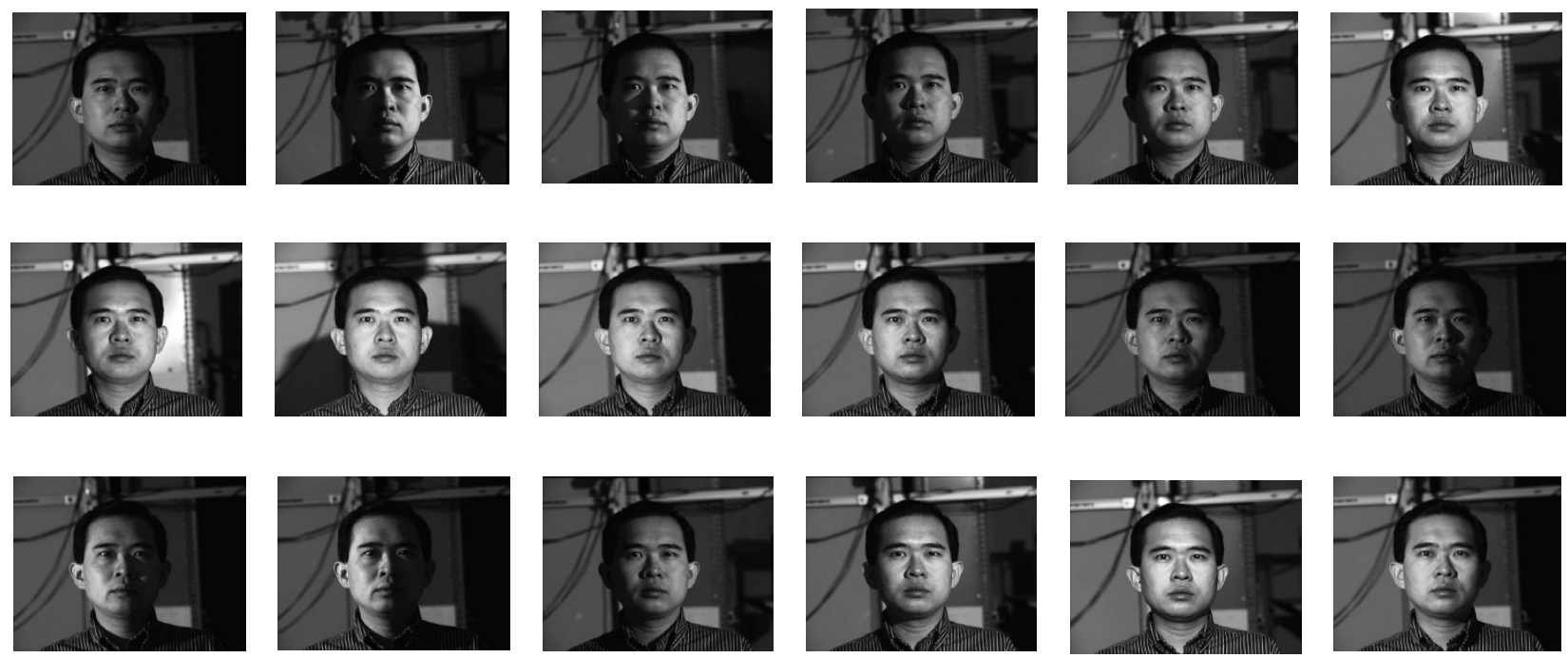

FIGURE 11: The 18 test images with different illumination conditions of a subject in the CMU PIE dataset.

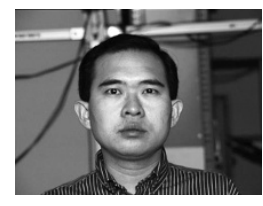

(a)

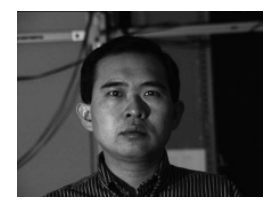

(b)

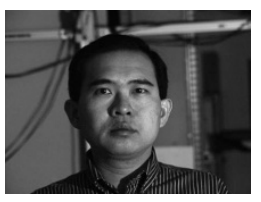

(c)

FIGURE 12: The original of three reference images of a subject in CMU PIE dataset: (a) 27_11 center light; (b) 27_13 left light; (c) 27_10 right light.

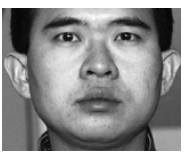

(a)

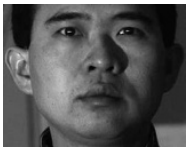

(b)

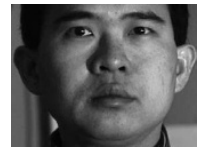

(c)
Figure 13: Three reference images of the same subject from Figure 12: (a) center light, (b) left light, (c) right light.

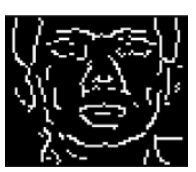

(a)

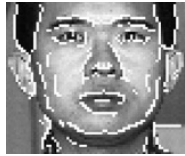

(b)

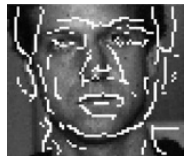

(c)
FIgure 14: (a) The edge contour of a subject in the CMU PIE dataset, (b) the edge contour of this subject is matched and overlaid onto his own face image, and (c) the edge contour of this subject is matched and overlaid onto another subject's face image.
TABLE 3: Comparison of recognition rates by using isotropic graylevel derivatives, 2D Gabor filter, eigenface, Fisherface, and the proposed robust image matching algorithm with three reference images on the CMU PIE Database.

\begin{tabular}{l|c}
\hline Methods & Three reference images \\
\hline Isotropic derivatives method & $58.87 \%$ \\
2D Gabor-Filter method & $73.47 \%$ \\
Eigenface & $83.49 \%$ \\
Fisherface & $88.55 \%$ \\
Proposed method & $94.78 \%$ \\
\hline
\end{tabular}

\section{ACKNOWLEDGMENT}

This work was jointly supported by the Program for Promoting Academic Excellence of Universities (89-E-FA04-14) and the National Science Council (project code 90-2213E-007-037), Taiwan. 


\section{REFERENCES}

[1] R. Chellappa, C. L. Wilson, and S. Sirohey, "Human and machine recognition of faces: a survey," Proceedings of the IEEE, vol. 83, no. 5, pp. 705-741, 1995.

[2] A. Pentland, "Looking at people: sensing for ubiquitous and wearable computing," IEEE Trans. on Pattern Analysis and Machine Intelligence, vol. 22, no. 1, pp. 107-119, 2000.

[3] A. Samal and P. A. Iyengar, "Automatic recognition and analysis of human faces and facial expressions: a survey," Pattern Recognition, vol. 25, no. 1, pp. 65-77, 1992.

[4] S. Belongie, J. Malik, and J. Puzicha, "Matching shapes," in Proc. 8th IEEE International Conference on Computer Vision (ICCV'01), vol. 1, pp. 454-461, Vancouver, British Columbia, Canada, July 2001.

[5] K. Hotta, T. Mishima, T. Kurita, and S. Umeyama, "Face matching through information theoretical attention points and its applications to face detection and classification," in Proc. 4th IEEE International Conference on Automatic Face and Gesture Recognition (FG '00), pp. 34-39, Grenoble, France, March 2000.

[6] R.-L. Hsu and A. K. Jain, "Face modeling for recognition," in Proc. International Conference on Image Processing (ICIP '01), vol. 2, pp. 693-696, Thessaloniki, Greece, October 2001.

[7] K. Sengupta and J. Ohya, "An affine coordinate based algorithm for reprojecting the human face for identification tasks," in Proc. International Conference on Image Processing (ICIP '97), vol. 3, pp. 340-343, Washington, DC, USA, October 1997.

[8] B. Takacs and H. Wechsler, "Face recognition using binary image metrics," in Proc. 3rd IEEE International Conference on Automatic Face and Gesture Recognition (FG '98), pp. 294-299, Nara, Japan, April 1998.

[9] C.-H. T. Yang, S.-H. Lai, and L.-W. Chang, "Robust face matching under different lighting conditions," in Proc. IEEE International Conference on Multimedia and Expo (ICME '02), vol. 2, pp. 149-152, Lausanne, Switzerland, August 2002.

[10] C.-H. T. Yang, S.-H. Lai, and L.-W. Chang, "An illuminationinsensitive face matching algorithm," in Proc. 3rd IEEE Pacific Rim Conference on Multimedia (PCM '02), pp. 1185-1192, Hsinchu, Taiwan, December 2002.

[11] J. Zhu, B. Liu, and S. C. Schwartz, "General illumination correction and its application to face normalization," in Proc. IEEE Int. Conf. Acoustics, Speech, Signal Processing (ICASSP '03), vol. 3, pp. 133-136, Hong Kong, China, April 2003.

[12] Y. Adini, Y. Moses, and S. Ullman, "Face recognition: the problem of compensating for changes in illumination direction," IEEE Trans. on Pattern Analysis and Machine Intelligence, vol. 19, no. 7, pp. 721-732, 1997.

[13] P. N. Belhumeur, J. P. Hespanha, and D. J. Kriegman, "Eigenfaces vs. Fisherfaces: recognition using class specific linear projection," IEEE Trans. on Pattern Analysis and Machine Intelligence, vol. 19, no. 7, pp. 711-720, 1997.

[14] K.-C. Chung, S. C. Kee, and S. R. Kim, "Face recognition using principal component analysis of Gabor filter responses," in Proc. International Workshop on Recognition, Analysis, and Tracking of Faces and Gestures in Real-Time Systems (RATFGRTS '99), pp. 53-57, Corfu, Greece, September 1999.

[15] A. S. Georghiades, D. J. Kriegman, and P. N. Belhumeur, "Illumination cones for recognition under variable lighting: faces," in Proc. IEEE Computer Society Conference on Computer Vision and Pattern Recognition (CVPR '98), pp. 52-58, Santa Barbara, Calif, USA, June 1998.
[16] A. S. Georghiades, P. N. Belhumeur, and D. J. Kriegman, "From few to many: illumination cone models for face recognition under variable lighting and pose," IEEE Trans. on Pattern Analysis and Machine Intelligence, vol. 23, no. 6, pp. 643660,2001

[17] P. Gros, "Color illumination models for image matching and indexing," in Proc. 15th International Conference on Pattern Recognition (ICPR '00), vol. 3, pp. 576-579, Barcelona, Spain, September 2000.

[18] C. Liu and H. Wechsler, "A Gabor feature classifier for face recognition," in Proc. 8th IEEE International Conference on Computer Vision (ICCV '01), vol. 2, pp. 270-275, Vancouver, British Columbia, Canada, July 2001.

[19] A. Mojsilovic and J. Hu, "Extraction of perceptually important colors and similarity measurement for image matching," in Proc. International Conference on Image Processing (ICIP '00), vol. 1, pp. 61-64, Vancouver, British Columbia, Canada, September 2000.

[20] B. Moghaddam, T. Jebara, and A. Pentland, "Bayesian face recognition," Pattern Recognition, vol. 33, no. 11, pp. 1771$1782,2000$.

[21] X. Mu, M. Artiklar, M. H. Hassoun, and P. Watta, "Training algorithms for robust face recognition using a templatematching approach," in Proc. International Joint Conference on Neural Networks (IJCNN'01), vol. 4, pp. 2877-2882, Washington, DC, USA, July 2001.

[22] M.-H. Yang, N. Ahuja, and D. Kriegman, "Face recognition using kernel eigenfaces," in Proc. International Conference on Image Processing (ICIP '00), vol. 1, pp. 37-40, Vancouver, British Columbia, Canada, September 2000.

[23] W. Y. Zhao and R. Chellappa, "Illumination-insensitive face recognition using symmetric shape-from-shading," in Proc. IEEE Conference on Computer Vision and Pattern Recognition (CVPR '00), vol. 1, pp. 286-293, Hilton Head Island, SC, USA, June 2000.

[24] G. J. Edwards, C. J. Taylor, and T. F. Cootes, "Interpreting face images using active appearance models," in Proc. 3rd IEEE International Conference on Automatic Face and Gesture Recognition (FG'98), pp. 300-305, Nara, Japan, April 1998.

[25] L. Wiskott, J.-M. Fellous, N. Kuiger, and C. von der Malsburg, "Face recognition by elastic bunch graph matching," IEEE Trans. on Pattern Analysis and Machine Intelligence, vol. 19, no. 7, pp. 775-779, 1997.

[26] D. A. Forsyth and J. Ponce, Computer Vision: A Modern Approach, Prentice-Hall, Upper Saddle River, NJ, USA, 2003.

[27] J. E. Dennis and R. B. Schnabel, Numerical methods for unconstrained optimization and nonlinear equations, Prentice-Hall, Upper Saddle River, NJ, USA, 1983.

[28] T. Sim, S. Baker, and M. Bsat, "The CMU pose, illumination, and expression database," IEEE Trans. on Pattern Analysis and Machine Intelligence, vol. 25, no. 12, pp. 1615-1618, 2003.

Chyuan-Huei Thomas Yang received the B.S. degree in mathematics from Tamkang University, Taipei County, Taiwan, in 1986, and the M.S. degree in computer science from the New Jersey Institute of Technology, Newark, New Jersey, USA, in 1992. He is a Ph.D. candidate in the Department of Computer Science, National Tsing Hua University, Hsinchu, Taiwan. His research interests include image processing, computer vision, pattern recognition, and face recognition.

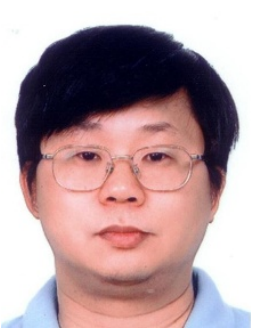


Shang-Hong Lai received the B.S. and M.S. degrees in electrical engineering from $\mathrm{Na}$ tional Tsing Hua University, Hsinchu, Taiwan, and the Ph.D. degree in electrical and computer engineering from the University of Florida, Gainesville, in 1986, 1988, and 1995, respectively. In 1995, he joined Siemens Corporate Research in Princeton, New Jersey, as a member of Technical Staff. Dr. Lai returned to Taiwan as a faculty

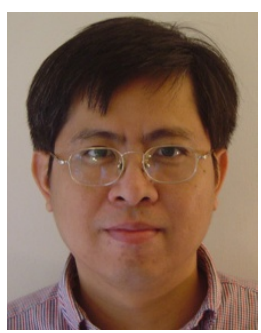
member in the Department of Computer Science, National Tsing Hua University, since 1999. He is currently an Associate Professor in the same department. His research interests include computer vision, image/video processing, medical imaging, and multimedia signal processing. He has authored more than 60 papers published in related international journals and conferences.

Long-Wen Chang received the B.S. degree in electrical engineering from National Cheng Kung University in 1976 and the M.S. and Ph.D. degrees in electrical engineering and computer engineering from the University of New Mexico in 1980 and 1984, respectively. In 1984, he joined Institute of Computer Science, National Tsing Hua University, Taiwan. His interests are in image processing, computer vision, com-

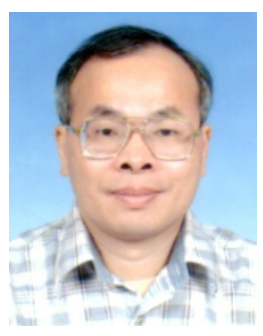
puter graphics, and information security and digital rights management. Dr. Chang is a Professor in the Department of Computer Science, National Tsing Hua University, Hsinchu, Taiwan. His works have been published in journals including "Computer Vision, Graphics, and Image Processing," "IEEE Transactions on Signal Processing," and "IEEE Transactions on Computer." Dr. Chang received an Excellent Research Award from the National Science Council. 